\title{
Toxicité de la chimiothérapie et des thérapies ciblées anticancéreuses
}

\section{sur la muqueuse buccale}

\author{
Guichard $\mathrm{M}^{1}$, Sibaud $\mathrm{V}^{2}$
}

${ }^{1}$ Service d'Odontologie, Centre de Lutte contre le cancer - Institut Claudius Regaud, Toulouse, France

${ }^{2}$ Service de Dermatologie, Centre de Lutte contre le cancer - Institut Claudius Regaud, Toulouse, France

La prise en charge de la maladie cancéreuse a été profondément bouleversée ces dernières années par le développement et l'utilisation croissante des nouvelles thérapies dites ciblées. Si ces nouvelles molécules, qui ciblent de façon plus spécifique des récepteurs et/ou des voies de signalisation surexprimées par les cellules cancéreuses, présentent un profil de tolérance le plus souvent correct, elles ne sont pas dénuées d'effets indésirables.

Du fait de leur expression physiologique dans la peau ou ses annexes et du rôle fondamental de certains d'entre eux dans l'homéostasie cutanée, la toxicité cutanéo-muqueuse induite par ces nouveaux traitements est souvent au premier plan (Sibaud 2011). On peut citer pour l'exemple la dépigmentation des phanères, le syndrome main-pied spécifique, la paronychie, l'éruption acnéiforme (Robert 2005) et, sur la muqueuse buccale, les ulcérations aphtoïdes, la réaction lichénoide ou l'ostéonécrose mandibulaire.

Cette présentation reprendra les principes fondamentaux des thérapies ciblées anticancéreuses et les principales toxicités cutanéo-muqueuses observées avec les thérapies ciblées, en détaillant plus spécifiquement celles liées aux inhibiteurs du récepteur à l'epidermal growth factor (erlotinib, cetuximab, lapatinib), aux inhibiteurs de l'angiogénèse (sorafenib, sunitinib, bevacizumab) ou de la voie mTOR (everolimus, temsirolimus). La toxicité sur la muqueuse buccale des chimiothérapies conventionnelles sera également abordée.

Correspondance : guichard.maryalis@claudiusregaud.fr 